\title{
Discontinuous Constituent Parsing with Pointer Networks
}

\author{
Daniel Fernández-González, Carlos Gómez-Rodríguez \\ Universidade da Coruña, CITIC \\ FASTPARSE Lab, LyS Group \\ Depto. de Ciencias de la Computación y Tecnologías de la Información \\ Elviña, 15071 A Coruña, Spain \\ \{d.fgonzalez, carlos.gomez\}@udc.es
}

\begin{abstract}
One of the most complex syntactic representations used in computational linguistics and NLP are discontinuous constituent trees, crucial for representing all grammatical phenomena of languages such as German. Recent advances in dependency parsing have shown that Pointer Networks excel in efficiently parsing syntactic relations between words in a sentence. This kind of sequence-to-sequence models achieve outstanding accuracies in building non-projective dependency trees, but its potential has not been proved yet on a more difficult task. We propose a novel neural network architecture that, by means of Pointer Networks, is able to generate the most accurate discontinuous constituent representations to date, even without the need of Part-of-Speech tagging information. To do so, we internally model discontinuous constituent structures as augmented non-projective dependency structures. The proposed approach achieves state-of-the-art results on the two widely-used NEGRA and TIGER benchmarks, outperforming previous work by a wide margin.
\end{abstract}

\section{Introduction}

Syntactic representations are increasingly being demanded by a wide range of artificial intelligence applications that process and understand natural language text or speech. This encourages the Natural Language Processing (NLP) community to develop more accurate and efficient parsers that are able to represent all complex grammatical phenomena present in human languages.

One of the most widely-used syntactic formalism is a constituent (or phrase-structure) representation. This describes the syntax of a sentence in terms of constituents or phrases and the hierarchical order between them, as shown in the constituent tree in Figure 1(a). As a simpler alternative, a dependency representation is also available. This straightforwardly connects each word of a sentence as a dependent of another, which acts as its head word. The resulting structure is a dependency tree like the one depicted in Figure 1(c).

Among constituent trees we can find the most informative syntactic representation currently available: discontinuous constituent trees. Apart from providing phrase-structure

Copyright (c) 2020, Association for the Advancement of Artificial Intelligence (www.aaai.org). All rights reserved. information, they extend regular or continuous constituent trees by allowing the representation of crossing branches and constituents with gaps in the middle, crucial for describing grammatical structures left out in the standard phrasestructure formalism, such as the free-word-order phenomena that can be found in languages such as German (see (Müller 2004) and references therein).

Unlike for regular constituent trees (Klein and Manning 2003), context-free grammars are not enough for deriving discontinuous structures and representing complex linguistic phenomena and, therefore, more expressive formalisms, such as Linear Context-Free Rewriting Systems (LCFRS) (Vijay-Shanker, Weir, and Joshi 1987), are required. However, due to the high complexity behind the generation of discontinuous phrase-structure representations, parsers based on probabilistic LCFRS, such as (Kallmeyer and Maier 2010), are not practical in terms of accuracy and speed. In the last decade, alternatives to tackle discontinuous constituent parsing with complex grammar-based approaches were presented.

On the one hand, one line of research proposes to extend transition-based parsers, which have been successfully applied for efficiently building continuous constituent trees (Zhu et al. 2013; Dyer et al. 2016), with transitions and data structures that are able to deal with discontinuity (Maier 2015; Stanojević and G. Alhama 2017; Coavoux, Crabbé, and Cohen 2019; Coavoux and Cohen 2019). This research branch currently yields the state of the art in discontinuous phrase-structure parsing.

In parallel, another stream led by (Hall and Nivre 2008; Versley 2014; Fernández-González and Martins 2015; Corro, Le Roux, and Lacroix 2017) proposes to represent discontinuous formalisms as dependencies with augmented information. This makes it possible to use efficient dependency parsers to perform the analysis and, after a recovery process, return a well-formed constituent tree. While they offer a similar efficiency as transition-based constituent parsers, they are currently slightly behind them in terms of accuracy. The main drawback of this approach is the large amount of complex labels necessary to encode the wide variety of syntactic structures into labelled dependency arcs, growing unbounded with the treebank size. This forces 
some authors, as (Fernández-González and Martins 2015; Corro, Le Roux, and Lacroix 2017), to use an extra module that, at a post-processing step after the parsing, labels each dependency to increase final accuracy. As a result, parsing and labelling tasks are learned and performed in a two-stage procedure. However, these two tasks might benefit from each other's information during training, as constituent information is encoded in both components (arc and label) so from an ideal standpoint, given good enough machine learning models, they should not be considered separately.

To address this problem, we propose a neural network architecture that, using internal augmented dependency representations based on (Fernández-González and Martins 2015) and following a multitask learning approach (Caruana 1997), can directly produce accurate discontinuous constituent trees in one step. Both tasks (creating dependencies and labeling them) are trained in parallel and using a shared representation, so that the whole model can jointly learn to predict the correct augmented labels that will finally produce well-formed phrase-structure trees.

In addition, our model relies on Pointer Networks (Vinyals, Fortunato, and Jaitly 2015) for creating dependency arcs between words of an input sentence. This kind of neural networks, obtained by modifying standard sequenceto-sequence models, yields remarkable accuracies in dependency parsing. In particular, they use attention (Bahdanau, Cho, and Bengio 2014) as a pointer to select positions from the input sequence, which can be easily adapted to connect words from an input sentence, as recently shown by (Ma et al. 2018; Fernández-González and Gómez-Rodríguez 2019). We adapt this state-of-the-art neural model to a harder task, also obtaining an outstanding performance. To the best of our knowledge, this is the first attempt that follows a sequence-to-sequence paradigm to perform discontinuous constituent parsing, without the need of any intermediate data structures to create partial trees as required by transition-based models previously cited.

To deal with the large amount of labels, we propose to use the biaffine classifier introduced by (Dozat and Manning 2017) that has shown a remarkable performance even in NLP taks with a more complex label scheme, such as Semantic Dependency Parsing (Dozat and Manning 2018). This shares the same representation as Pointer Networks and is jointly trained to produce well-formed discontinuous constituent trees in a single step.

The resulting neural model ${ }^{1}$ produces the most accurate discontinuous constituent representations reported so far. We conduct experiments on both widely-known NEGRA (Skut et al. 1997) and TIGER (Brants et al. 2002) treebanks, which contain a large number of German sentences syntactically annotated by constituent trees with a high degree of discontinuity: the prevalence of discontinuous constituent structures is over 25\% in both treebanks (Maier and Lichte 2011). In both benchmarks, our approach achieves accuracies beyond 85\% F-score (even without Part-of-Speech (POS) tagging information), surpassing the current state of the art by a wide margin without the need of orthogonal tech-

\footnotetext{
${ }^{1}$ Available at https://github.com/danifg/DiscoPointer
}

niques such as re-ranking or semi-supervision.

\section{Modelling Discontinuous Constituent Trees Preliminaries}

Let $w_{1}, w_{2}, \ldots, w_{n}$ be a sentence, where $w_{i}$ denotes a word in the $i$ th position. A constituent tree is a tree with the $n$ words of the sentence as leaf nodes, and phrases (or constituents) as internal nodes. Each constituent can be represented as a tuple $\left(X, \mathcal{C}, w_{h}\right)$, where $X$ is the non-terminal symbol, $\mathcal{C}$ is the yield, i.e. the set of words $w_{i}$ included in its span, and $h$ is the word in $\mathcal{C}$ that acts as head. A language-specific handwritten set of rules is used to select the head word. For instance, the head word of the constituent VROOT in Figure 1(a) is the word $\mathrm{kam}$ in constituent $S$.

Given a tree, if the yield $(\mathcal{C})$ of each of its constituents is a continuous substring of the sentence, then we say that the tree is continuous. Otherwise, the tree is classified as discontinuous, meaning that we can find at least one constituent with a span that is interrupted by one or more gaps between its words. For instance, in Figure 1(a), the span of constituent (NP, $\{$ Es, nichts, Interessantes $\}$, Interessantes) is interrupted by the word kam from a different constituent, generating crossing branches. Finally, if there are no constituents with only one child node, we call it a unaryless constituent tree.

On the other hand, a dependency tree is a directed tree spanning all the words $w_{i}$ with $i$ ranging from 1 to $n$. Each dependency arc can be represented as $\left(w_{h}, w_{d}, l\right)$, with $w_{h}$ being the head word of the dependent word $w_{d}$ (with $h$, $d \in[1, n])$ and $l$ being the dependency label indicating the syntactic role played by $w_{d}$. If, for every dependency arc $\left(w_{h}, w_{d}, l\right)$, there is a directed path from $w_{h}$ to all words $w_{i}$ between words $w_{h}$ and $w_{d}$, we classify it as a projective dependency tree. If not, it is described as non-projective, resulting in a tree with crossing arcs as the one depicted in Figure 1(c).

It can be noticed from these definitions that, in order to represent the same syntactic phenomenon as described in a discontinuous constituent tree, we will need to use a nonprojective dependency structure in order to handle discontinuities. However, a constituent tree is able to provide information that cannot be represented in a regular dependency tree (Kahane and Mazziotta 2015).

\section{Constituents as Augmented Dependencies}

Following (Fernández-González and Martins 2015), we decompose a discontinuous unariless phrase-structure tree into a set of non-projective dependency arcs with enriched information. This allows us to use a non-projective dependency parser to efficiently perform discontinuous phrase-structure parsing.

A constituent tree with $m$ words can be decomposed into a set of $m$ spines (Carreras, Collins, and Koo 2008), one per word, as shown in Figure 1(b) for the discontinuous tree in Figure 1(a). These spines and their interaction to finally build a constituent tree can be represented in an augmented dependency tree as described by (FernándezGonzález and Martins 2015). More in detail, they propose 


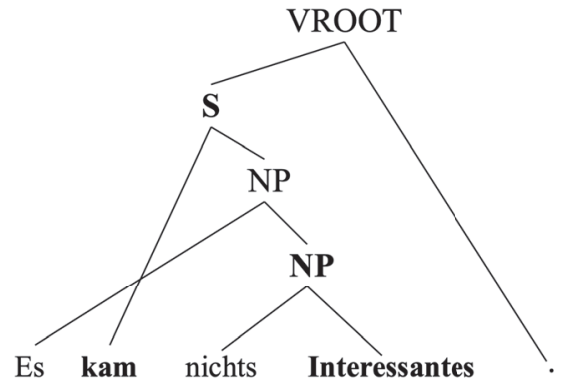

a) Discontinuous constituent tree.

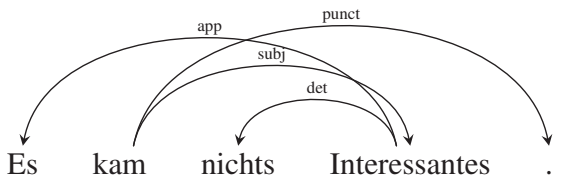

c) Unlabelled dependency tree.

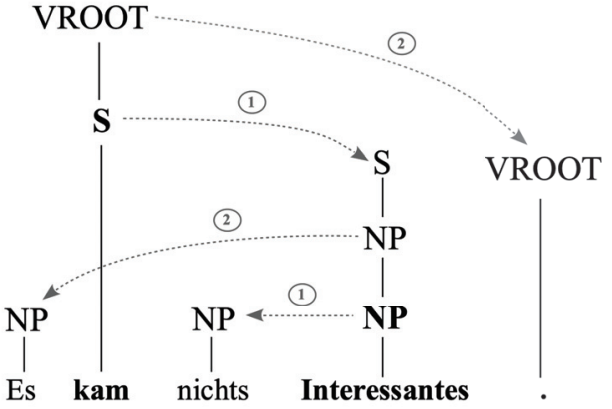

b) Spines interaction.

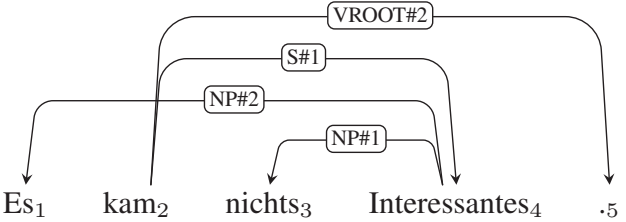

d) Augmented dependency tree.

Figure 1: Augmented dependency representation of a sentence taken from NEGRA corpus in discontinuous constituent format, as well as the spine interaction required for its reconstruction. Head nodes of each constituent are marked in bold.

to encode each constituent into dependencies: for each constituent $\left(X, \mathcal{C}, w_{h}\right)$, each child node, that can be a word $w_{d}$ or a constituent $\left(Y, \mathcal{G}, w_{d}\right)$ with $w_{d} \neq w_{h}$, is encoded into an unlabelled dependency arc with the form $\left(w_{h}, w_{d}\right)$. Basically, each non-head child node is attached to the head word and, if the involved child node is a constituent, its own head word is used to build the dependency arc.

Additionally, to represent the original phrase structure it is also necessary to save some vital information into arc labels: the non-terminal symbol $X$ plus an index that indicates the order in which spines are attached. This index will be crucial in those cases where more than one constituent share the same head word (and, therefore, the same head spine), but they are at a different level in the original tree and, therefore, should be created in a different hierarchical order. The final dependency arcs will have the form $\left(w_{h}, w_{i}, X \# p\right)$, where $p$ is the attachment order. For instance, constituent (NP, \{nichts, Interessantes $\}$, Interessantes) in Figure 1(a), is encoded as the augmented dependency arc (Interessantes, nichts, NP\#1) in Figure 1(d), and constituent (NP, $\{$ Es, nichts, Interessantes $\}$, Interessantes) is represented with (Interessantes, Es, NP\#2). Both constituents share the same head spine anchored to word Interessantes, but they are attached in a different level.

To recover the original discontinuous constituent tree, we just have to follow the steps provided by the augmented dependencies from a spine-based perspective. Dependencies will indicate which spines are involved in the attachment and the direction of the spine interaction (head and dependent roles); and, on the other hand, labels will describe in what order dependent spines are attached to the head spine, as well as the non-terminal symbol of the resulting constituent. For instance, the augmented dependency tree in Figure 1(d) describes the spine interaction depicted in Figure 1(b). In the example, we can see that the dependent spines anchored to words Es and nichts are attached in different order and level from the head spine lexicalized by the word Interessantes. Without the attachment order, all dependent spines would be attached at the same level and the resulting constituent would be a different structure: in the example, a single flat NP phrase with the three words as its child nodes.

It is also worth mentioning that the above described dependency-based representation is not able to encode unary constituents and, as a consequence, this information will be lost after the constituent tree is recovered. However, as stated by (Fernández-González and Martins 2015), unary nodes are very uncommon in discontinuous representations: for instance, the NEGRA treebank has no unaries at all and, for the TIGER dataset, the fraction of unaries is around $1 \%$. Therefore, we do not perform unary recovery in a postprocessing step and simply apply a non-projective dependency parser on the resulting augmented trees.

\section{Neural Network Architecture}

We use a multi-task learning strategy to train in parallel both the Pointer Network, in charge of connecting words, and the labeler, a multiclass classifier in charge of tagging each dependency arc with the augmented information, to finally build a well-formed discontinuous constituent tree. In the next subsections, we describe the proposed pointer-networkbased architecture for labelled dependency parsing.

\section{Pointer Networks}

(Vinyals, Fortunato, and Jaitly 2015) introduced a novel neural architecture, called Pointer Network, that can be seen as a variation of standard sequence-to-sequence models. The proposed neural network can learn the conditional probability of an output sequence of discrete numbers that correspond to positions from the input sequence, with input 
length being variable. They propose to use a mechanism of neural attention (Bahdanau, Cho, and Bengio 2014) to select positions from the input, without requiring a fixed size of the output dictionary. Thanks to that, Pointer Networks are suitable for addressing those problems where the target classes considered at each step are variable and depend on the length of the input sequence.

Unlabelled dependency parsing is one of the tasks where Pointer Networks can be easily applied: the input is a (variable-length) sequence of words from a sentence and the output is a sequence of numbers that correspond to the positions of the assigned head words. In (Fernández-González and Gómez-Rodríguez 2019), we can find a transition-based perspective of this idea, but it can be also defined as a purely sequence-to-sequence approach, as, unlike in classic transition-based dependency parsers (Nivre 2003), neither transitions nor data structures are required.

We follow the convention of representing scalars in lowercase italics $(i)$, vectors in lowercase bold $(\mathbf{v})$, matrices in uppercase italics $(M)$, and higher order tensors in uppercase bold (T).

Encoder Let $w_{1}, w_{2}, \ldots, w_{n}$ be an input sentence. The encoder of our network relies on a BiLSTM-CNN architecture, developed by (Ma and Hovy 2016), to encode each word $w_{i}$ into an encoder hidden state $\mathbf{h}_{i}$.

Convolutional Neural Networks (CNN) (LeCun et al. 1989) are used for extracting character-level representations of words. In particular, for each word $w_{i}$, the $\mathrm{CNN}$ receives a concatenation of embedding vectors of each character as input and produces a character-level representation $\mathbf{e}_{i}^{c}$. Then, each word $w_{i}$ is represented by the concatenation of three embeddings: character-level word embedding $\mathbf{e}_{i}^{c}$, pretrained word embedding $\mathbf{e}_{i}^{w}$ and randomly initialized part-of-speech (POS) tag embedding $\mathbf{e}_{i}^{p}(\oplus$ stands for concatenation):

$$
\mathbf{x}_{i}=\mathbf{e}_{i}^{c} \oplus \mathbf{e}_{i}^{w} \oplus \mathbf{e}_{i}^{p}
$$

In order to attach every word from the sentence to another, we need to add a dummy root node $w_{0}$ (represented with a special vector $\mathrm{x}_{0}$ ) at the beginning of the sentence. This will be linked as the head of the real syntactic root word.

After that, the resulting embedding representation $\mathbf{x}_{i}$ of each word $w_{i}$ is fed one-by-one into a Bi-directional Long Short-Term Memory (BiLSTM) (Graves and Schmidhuber 2005) that captures context information in both directions and generates a vector representation $\mathbf{h}_{i}$ :

$$
\mathbf{h}_{i}=\mathbf{h}_{l i} \oplus \mathbf{h}_{r i}=\operatorname{BiLSTM}\left(\mathbf{x}_{i}\right)
$$

Decoder A unidirectional LSTM (Hochreiter and Schmidhuber 1997) is used as a decoder that, at each time step, uses the encoder hidden state $\mathbf{h}_{i}$ of the word $w_{i}$, currently being processed, as input and outputs a decoder hidden state $\mathbf{s}_{t}$. To add extra information to the input of the LSTM, we follow (Fernández-González and Gómez-Rodríguez 2019) and add hidden states of previous $\left(\mathbf{h}_{i-1}\right)$ and next $\left(\mathbf{h}_{i+1}\right)$ words. As proposed by (Ma et al. 2018), we use element-wise sum of the three hidden states, instead of concatenating them, in order to not increase the dimension of the resulting input vector $\mathbf{r}_{i}$ :

$$
\mathbf{r}_{i}=\mathbf{h}_{i-1}+\mathbf{h}_{i}+\mathbf{h}_{i+1}
$$

$$
\mathbf{s}_{t}=\operatorname{LSTM}\left(\mathbf{r}_{i}\right)
$$

Once $\mathbf{s}_{t}$ is generated from $\mathbf{r}_{i}$, the attention vector $\mathbf{a}^{t}$ needs to be computed as follows:

$$
\begin{aligned}
& \mathbf{v}_{j}^{t}=\operatorname{score}\left(\mathbf{s}_{t}, \mathbf{h}_{j}\right) \\
& \mathbf{a}^{t}=\operatorname{softmax}\left(\mathbf{v}^{t}\right)
\end{aligned}
$$

where an attention scoring function (score ()$)$ is used to compute scores between $\mathbf{s}_{t}$ (the decoder representation of the word $w_{i}$ currently being processed) and each encoder hidden state $\mathbf{h}_{j}$ from the input. Then, a softmax is applied on the resulting score vector $\mathbf{v}^{t}$ to compute a probability distribution over the input. The generated attention vector $\mathbf{a}^{t}$ is used as a pointer to select the highest-scoring position $j$ from the input. The pointed word $w_{j}$ is attached as head of word $w_{i}$, currently being processed, building an unlabeled dependency arc $\left(w_{j}, w_{i}\right)$. In case the arc $\left(w_{j}, w_{i}\right)$ generates a cycle in the already-built dependency graph, we select the next highest-scoring position from the input pointed by $\mathbf{a}^{t}$.

The decoding process starts in word $w_{1}$ and it is repeated word-by-word from left to right until the last one $\left(w_{n}\right)$ is attached, requiring just $n$ steps to fully parse the input sentence. Therefore, since cycles can be checked in amortized linear time $(O(n))$ and, at each step, the attention vector $\mathbf{a}^{t}$ must be computed over the input, the overall runtime complexity is $O\left(n^{2}\right)$. Figure 2 depicts the neural architecture and the decoding procedure for the dependency structure in Figure $1(\mathrm{~d})$.

Attention Mechanism As attention score function, we follow (Ma et al. 2018) and adopt the biaffine attention mechanism, developed by (Dozat and Manning 2017), to compute score vector $\mathbf{v}^{t}$ :

$$
\mathbf{v}_{j}^{t}=\mathbf{s}_{t}^{T} W \mathbf{h}_{j}+\mathbf{U}^{T} \mathbf{s}_{t}+\mathbf{V}^{T} \mathbf{h}_{j}+\mathbf{b}
$$

where parameters $W$ is the weight matrix of the bi-linear term, $\mathbf{U}$ and $\mathbf{V}$ are the weight tensors of the linear terms and $\mathbf{b}$ is the bias vector. Basically, the bi-linear term evaluates the score of assigning the word $w_{i}$ (represented by $\mathbf{s}_{t}$ ) to the head word $w_{j}$ from the input, and the two linear terms compute the scores of both words considered independently.

Finally, in order to reduce the dimensionality and overfitting of the neural model, a multilayer perceptron (MLP) is used to transform the hidden representations $\mathbf{h}_{j}$ and $\mathbf{s}_{t}$ before the attention score function is applied, as discussed by (Dozat and Manning 2017).

\section{Biaffine labeler}

We follow (Dozat and Manning 2017) and implement the labeler as a biaffine multi-class classifier. Unlike them, we use the previously described BiLSTM-CNN architecture as encoder.

(Dozat and Manning 2017) relies on a biaffine attention mechanism as scoring function for computing the score of assigning a label $l$ on a predicted arc between the dependent word $w_{i}$ and the head word $w_{j}$, assigning to that arc the highest-scoring label. In particular, the encoder hidden state $\mathbf{h}_{j}$ and the decoder hidden state $\mathbf{s}_{t}$ (representing $w_{j}$ and $w_{i}$, 


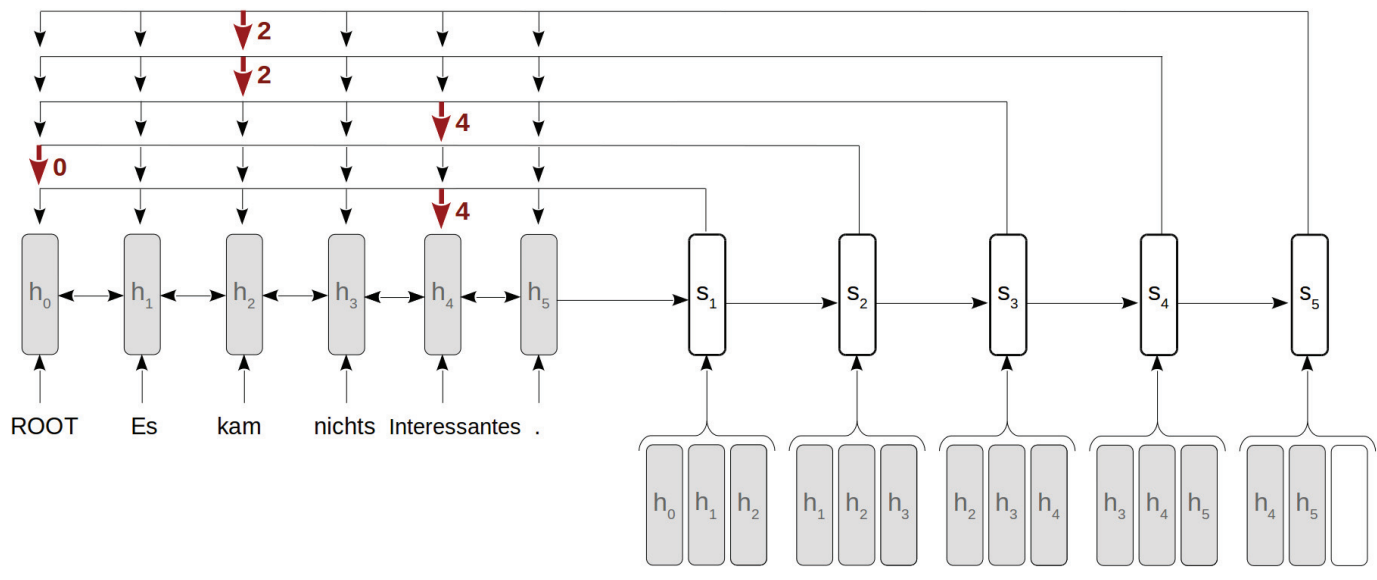

Figure 2: Pointer Network architecture and decoding steps to output the dependency tree in Figure 1(d).

respectively) are used as input to obtain the score $\mathbf{s}_{t j}^{l}$ for each label $l$ as shown in the following equation:

$$
\mathbf{s}_{t j}^{l}=\mathbf{s}_{t}^{T} W_{l} \mathbf{h}_{j}+\mathbf{U}_{l}^{T} \mathbf{s}_{t}+\mathbf{V}_{l}^{T} \mathbf{h}_{j}+\mathbf{b}_{l}
$$

where a distinct weight matrix $W_{l}$, weight tensors $\mathbf{U}_{l}$ and $\mathbf{V}_{l}$ and bias $\mathbf{b}_{l}$ are used for each label $l$, where $l \in\{1,2, \ldots, L\}$ and $L$ is the number of labels. The first term on the right side of the equation represents the score of assigning the label $l$ to the arc between dependent $w_{i}$ and head $w_{j}$; and the second and third terms express the score of the label $l$ when the dependent and head are considered independently. Please note that a MLP is also used to transform hidden state vectors $\mathbf{h}_{j}$ and $\mathbf{s}_{t}$, before feeding the biaffine classifier, in order to filter not relevant information and, as a consequence, reduce model dimensionality (Dozat and Manning 2017).

\section{Multitask Learning}

Our goal is to provide a fully-parsed non-projective dependency tree in a single stage, while the parsing and the labelling should be individually undertaken due to the large amount of labels resulting from the formalism by (Fernández-González and Martins 2015). We follow a multitask learning strategy (Caruana 1997) to achieve that: a single neural architecture is trained for more than one task, sharing a common representation and benefiting from each other.

In particular, the labeler shares the same encoder as the parser, providing a common encoder hidden state representation for both components. As stated by (Kiperwasser and Goldberg 2016), training the BiLSTM-CNN encoder to correctly predict dependency labels significantly improves unlabelled parsing accuracy and vice versa. This is specially crucial in our approach where a constituent structure is jointly encoded in dependency arcs and labels, and a wrong label can lead to a completely different phrase-structure tree after the recovering.

In addition, the labeler and the parser are simultaneously trained by optimizing the sum of their objectives. On the one hand, a dependency tree $y$ for an input sentence of length $n$ is decomposed into a set of $n$ directed $\operatorname{arcs} a_{1}, \ldots, a_{n}$, where each $\operatorname{arc} a_{i}$ is represented by the head word $w_{h}$ and the dependent word $w_{i}$ in position $i$ in the sentence. Therefore, to train the parser, we factorize the conditional probability $P_{\theta}(y \mid x)$ of a dependency tree $y$ for a sentence $x$ into a set of head-dependent pairs $\left(w_{h}, w_{i}\right)$ as follows:

$$
P_{\theta}(y \mid x)=\prod_{i=1}^{n} P_{\theta}\left(a_{i} \mid a_{<i}, x\right)=\prod_{i=1}^{n} P_{\theta}\left(w_{h} \mid w_{i}, a_{<i}, x\right)
$$

Then, the unlabelled parsing model is trained by minimizing the negative log likelihood of choosing the correct head word $w_{h}$ for the word $w_{i}$ currently being processed, given the previous predicted arcs $a_{<i}$. This is implemented as cross-entropy loss:

$$
\mathcal{L}_{\text {arc }}=-\log P_{\theta}\left(w_{h} \mid w_{i}, a_{<i}, x\right)
$$

On the other hand, the biaffine labeler is trained by minimizing the negative log probability of assigning the correct label $l$, given a dependency arc with head word $w_{h}$ and dependent word $w_{i}$. A cross entropy loss $\mathcal{L}_{\text {label }}$ for dependency label prediction is computed when training:

$$
\mathcal{L}_{\text {label }}=-\log P_{\theta}\left(l \mid w_{h}, w_{i}\right)
$$

Finally, we train a joint model by summing the losses $\mathcal{L}_{\text {arc }}$ and $\mathcal{L}_{\text {label }}$ prior to computing the gradients. In that way, model parameters are learned to minimize the sum of the cross-entropy loss objectives over the whole corpus.

\section{Experiments}

\section{Data}

We test our new approach on two widely-used discontinuous German treebanks: NEGRA (Skut et al. 1997) and TIGER (Brants et al. 2002). For the latter, we use the split provided in the SPMRL14 shared task (Crabbé 2014), and, for NEGRA, we follow the standard splits (Dubey and Keller 2003). While in TIGER we use the predicted POS tags provided in the shared task, we run TurboTagger (Martins, 


\begin{tabular}{lc}
\hline Architecture hyper-parameters & \\
\hline CNN window size & 3 \\
CNN number of filters & 50 \\
BiLSTM encoder layers & 3 \\
BiLSTM encoder size & 512 \\
LSTM decoder layers & 1 \\
LSTM decoder size & 512 \\
POS tag/word/character embedding dimension & 100 \\
LSTM layers/embeddings dropout & 0.33 \\
MLP layers & 1 \\
MLP activation function & ELU \\
Arc MLP size & 512 \\
Label MLP size & 128 \\
\hline Adam optimizer hyper-parameters & \\
\hline Initial learning rate & 0.001 \\
$\beta_{1}, \beta_{2}$ & 0.9 \\
Decay rate & 0.75 \\
Gradient clipping & 5.0 \\
\hline
\end{tabular}

Table 1: Model hyper-parameters.

Almeida, and Smith 2013) for predicting POS tags for NEGRA. Finally, for both treebanks, we apply the head-rule sets by (Rehbein 2009) to identify head words in constituent structures and build the equivalent augmented dependency trees. Following standard practice, we use discodop ${ }^{2}$ (van Cranenburgh, Scha, and Bod 2016) with the configuration file proper.prm for evaluation. This ignores punctuation and root symbols.

\section{Settings}

We use the Adam optimizer (Kingma and Ba 2014) and follow (Ma et al. 2018; Dozat and Manning 2017) for parameter optimization and hyper-parameter selection. These are detailed in Table 1. All embeddings are fine-tuned during training and, for initializing word vectors, we use the pretrained structured-skipgram embeddings developed by (Ling et al. 2015). Due to random initializations, we report average accuracy over 5 repetitions for each experiment tested. In addition, during training, the model with the highest Labelled Attachment Score (LAS) on the augmented dependency version of the development set is chosen. Finally, we use 10-beam-search decoding for all experiments.

\section{Results}

In Table 2, we report the overall F-score on labeled constituents and a specific F-score measured only on discontinuous constituents (Disc. F1) of current state-of-the-art parsers in comparison to our neural model.

As seen in the table, the proposed neural model is able to outperform all existing systems by a wide margin in both predicted- and gold-POS-tagging scenarios. While gold POS tags prove to be beneficial for the parsing process, using predicted ones yields the same performance as not providing POS-tagging information at all. Finally, we can also

\footnotetext{
${ }^{2}$ https://github.com/andreasvc/disco-dop
}

conclude from these results that, even without neither pretrained word embeddings nor POS tags, our approach still surpasses the current state of the art by a wide margin.

\section{Related work}

(Hall and Nivre 2008) was the first work that reduced discontinuous constituent trees into a non-projective dependency representation so that the advances in dependency parsing could be easily adapted to build phrase-structure trees. The main weakness of this first attempt was the spine enconding scheme. They encoded the whole dependent spines (compounded by one or more non-terminal symbols) plus the attachment position in the head spine into complex arc labels, leading to a combinatorial explosion and a significantly large number of labels. This considerably harmed final parsing performance. Following the same encoding scheme, (Versley 2014) introduced an easy-first approach that improved accuracy and speed, but the complex label scheme was still penalizing parsing accuracy.

(Fernández-González and Martins 2015) presented a lighter encoding strategy. They proposed to encode spine interactions by just saving the non-terminal node that results from the spine attachment and the position where it is undertaken. Unlike the previous complex scheme, this cannot deal with unary constituents that should be recovered as a postprocessing step. In addition, they used a pre-deep-learning parser, Turboparser (Martins, Almeida, and Smith 2013), that not only reported parsing accuracies notably below the current state-of-the-art, but also was not able to perform a competitive labeling due to the still high number of labels. As a solution, they just performed labeling as a separate step after the parsing was done. The resulting approach notably boosted final accuracy.

The latest attempt to deal with discontinuous phrasestructure parsing as a dependency problem was (Corro, Le Roux, and Lacroix 2017). Based on the work by (Carreras, Collins, and Koo 2008), that shows how lexicalized spinal TAGs (Tree Adjoining Grammars) can be used to perform continuous constituent parsing, they proposed a variant for discontinuous structures. Their approach works by reducing spinal TAG parsing to a maximum spanning tree problem and using a neural graph-based dependency parser (Dozat and Manning 2017) to solve it. To simplify the process, they also perform labeling after dependency trees are built.

In our approach, we follow the simplest grammaragnostic encoding scheme introduced by (FernándezGonzález and Martins 2015) and develop a state-of-the-art neural network architecture that is able to produce an output in a single step by jointly learning to create and label augmented dependency arcs. We consider that this one-stage approach is crucial since arcs and labels are strongly interdependent and they jointly encode the constituent structure.

\section{Conclusions}

We present a novel neural architecture that is able to produce the most accurate discontinuous constituent representations reported so far on the two main benchmarks: NE- 


\begin{tabular}{lcccc} 
& \multicolumn{2}{c}{ NEGRA } & \multicolumn{2}{c}{ TIGER } \\
Parser & F1 & Disc. F1 & F1 & Disc. F1 \\
\hline Predicted POS tags & & & & \\
Fernández-González and Martins (2015) & 77.0 & & 77.3 & \\
van Cranenburgh, Scha, and Bod (2016), $\leq 40$ & 74.8 & & 79.5 & \\
Versley (2016) & & & 77.0 & \\
Stanojević and G. Alhama (2017) & & & 79.3 & \\
Coavoux and Crabbé (2017) & & 75.1 & \\
Gebhardt (2018) & 83.2 & 54.6 & 82.7 & 55.9 \\
Coavoux, Crabbé, and Cohen (2019) & 83.2 & 56.3 & 82.5 & 55.9 \\
Coavoux and Cohen (2019) & $\mathbf{8 5 . 4}$ & $\mathbf{5 8 . 8}$ & $\mathbf{8 5 . 3}$ & $\mathbf{5 9 . 1}$ \\
This work & & & & \\
Gold POS tags & 77.0 & 19.8 & 74.7 & 18.8 \\
Maier (2015) & 80.5 & & 80.6 & \\
Fernández-González and Martins (2015) & & & 76.5 & \\
Maier and Lichte (2016) & & & 81.6 & \\
Corro, Le Roux, and Lacroix (2017) & 82.9 & & 81.6 & \\
Stanojević and G. Alhama (2017) & 82.2 & 50.0 & 81.6 & 49.2 \\
Coavoux and Crabbé (2017) & $\mathbf{8 6 . 1}$ & $\mathbf{5 9 . 9}$ & $\mathbf{8 6 . 3}$ & $\mathbf{6 0 . 7}$ \\
This work & & & & \\
This work & 85.7 & 58.6 & 85.7 & 60.4 \\
$\quad$ w/o POS tags & 83.7 & 54.7 & 84.6 & 57.9 \\
\hline w/o POS tags, w/o pre-trained word embs. & & &
\end{tabular}

Table 2: Accuracy comparison of state-of-the-art discontinuous constituent parsers on NEGRA and TIGER.

GRA and TIGER treebanks. We rely on Pointer Networks (Vinyals, Fortunato, and Jaitly 2015) and a biaffine classifier (Dozat and Manning 2017) to efficiently generate discontinuous phrase-structure trees. The proposed grammar- and data-structure-agnostic neural model is able to accurately process the large amount of labels generated by the formalism introduced by (Fernández-González and Martins 2015) and, unlike the original work, provides an output in a onestage procedure. In addition, our approach is orthogonal to other techniques such as re-ranking or semi-supervision that can certainly boost final accuracy.

\section{Acknowledgments}

This work has received funding from the European Research Council (ERC), under the European Union's Horizon 2020 research and innovation programme (FASTPARSE, grant agreement No 714150), from the ANSWER-ASAP project (TIN2017-85160-C2-1-R) from MINECO, and from Xunta de Galicia (ED431B 2017/01).

\section{References}

Bahdanau, D.; Cho, K.; and Bengio, Y. 2014. Neural machine translation by jointly learning to align and translate. CoRR abs/1409.0473.

Brants, S.; Dipper, S.; Hansen, S.; Lezius, W.; and Smith, G. 2002. TIGER treebank. In Proceedings of the 1st Workshop on Treebanks and Linguistic Theories (TLT), 24-42.

Carreras, X.; Collins, M.; and Koo, T. 2008. Tag, dynamic programming, and the perceptron for efficient, feature-rich parsing. In Proceedings of the Twelfth Conference on Computational Natural Language Learning (CoNLL), 9-16.

Caruana, R. 1997. Multitask learning. Mach. Learn. 28(1):41-75. Coavoux, M., and Cohen, S. B. 2019. Discontinuous constituency parsing with a stack-free transition system and a dynamic oracle.
In Proceedings of the 2019 Conference of the North American Chapter of the Association for Computational Linguistics, 204217. Minneapolis, Minnesota: Association for Computational Linguistics.

Coavoux, M., and Crabbé, B. 2017. Incremental discontinuous phrase structure parsing with the GAP transition. In Proceedings of the 15th Conference of the European Chapter of the Association for Computational Linguistics, 1259-1270. Valencia, Spain: Association for Computational Linguistics.

Coavoux, M.; Crabbé, B.; and Cohen, S. B. 2019. Unlexicalized transition-based discontinuous constituency parsing. Transactions of the Association for Computational Linguistics 7:73-89.

Corro, C.; Le Roux, J.; and Lacroix, M. 2017. Efficient discontinuous phrase-structure parsing via the generalized maximum spanning arborescence. In Proceedings of the 2017 Conference on Empirical Methods in Natural Language Processing, 1644-1654. Copenhagen, Denmark: Association for Computational Linguistics.

Crabbé, B. 2014. Multilingual discriminative shift-reduce phrase structure parsing for the spmrl 2014 shared task.

Dozat, T., and Manning, C. D. 2017. Deep biaffine attention for neural dependency parsing. In ICLR. OpenReview.net.

Dozat, T., and Manning, C. D. 2018. Simpler but more accurate semantic dependency parsing. In Proceedings of the 56th Annual Meeting of the Association for Computational Linguistics, 484490. Melbourne, Australia: Association for Computational Linguistics.

Dubey, A., and Keller, F. 2003. Probabilistic parsing for German using sister-head dependencies. In Proceedings of the 41st Annual Meeting of the Association for Computational Linguistics, 96-103. Sapporo, Japan: Association for Computational Linguistics.

Dyer, C.; Kuncoro, A.; Ballesteros, M.; and Smith, N. A. 2016. Recurrent neural network grammars. In HLT-NAACL, 199-209. The Association for Computational Linguistics. 
Fernández-González, D., and Gómez-Rodríguez, C. 2019. Left-toright dependency parsing with pointer networks. In Proceedings of the 2019 Conference of the North American Chapter of the Association for Computational Linguistics, 710-716. Minneapolis, Minnesota: Association for Computational Linguistics.

Fernández-González, D., and Martins, A. F. T. 2015. Parsing as reduction. In Proceedings of the 53rd Annual Meeting of the Association for Computational Linguistics), 1523-1533. Beijing, China: Association for Computational Linguistics.

Gebhardt, K. 2018. Generic refinement of expressive grammar formalisms with an application to discontinuous constituent parsing. In Proceedings of the 27th International Conference on Computational Linguistics, 3049-3063. Santa Fe, New Mexico, USA: Association for Computational Linguistics.

Graves, A., and Schmidhuber, J. 2005. Framewise phoneme classification with bidirectional lstm and other neural network architectures. Neural Networks 18(5):602 - 610. IJCNN 2005.

Hall, J., and Nivre, J. 2008. Parsing discontinuous phrase structure with grammatical functions. In Proceedings of the 6th International Conference on Advances in Natural Language Processing, GoTAL '08, 169-180. Berlin, Heidelberg: Springer-Verlag.

Hochreiter, S., and Schmidhuber, J. 1997. Long short-term memory. Neural Comput. 9(8):1735-1780.

Kahane, S., and Mazziotta, N. 2015. Syntactic polygraphs. a formalism extending both constituency and dependency. In Proceedings of the 14th Meeting on the Mathematics of Language (MoL 2015), 152-164. Chicago, USA: Association for Computational Linguistics.

Kallmeyer, L., and Maier, W. 2010. Data-driven parsing with probabilistic linear context-free rewriting systems. In Proceedings of the 23rd International Conference on Computational Linguistics (Coling 2010), 537-545. Beijing, China: Coling 2010 Organizing Committee.

Kingma, D. P., and Ba, J. 2014. Adam: A method for stochastic optimization. Published as a conference paper at the 3rd International Conference for Learning Representations, San Diego, 2015.

Kiperwasser, E., and Goldberg, Y. 2016. Simple and accurate dependency parsing using bidirectional LSTM feature representations. TACL 4:313-327.

Klein, D., and Manning, C. D. 2003. Accurate unlexicalized parsing. In Proceedings of the 41st Annual Meeting of the Association for Computational Linguistics (ACL), 423-430.

LeCun, Y.; Boser, B.; Denker, J. S.; Henderson, D.; Howard, R. E.; Hubbard, W.; and Jackel, L. D. 1989. Backpropagation applied to handwritten zip code recognition. Neural Comput. 1(4):541-551.

Ling, W.; Dyer, C.; Black, A. W.; and Trancoso, I. 2015. Two/too simple adaptations of Word2 Vec for syntax problems. In Proceedings of the 2015 Conference of the North American Chapter of the Association for Computational Linguistics, 1299-1304. Denver, Colorado: Association for Computational Linguistics.

Ma, X., and Hovy, E. 2016. End-to-end sequence labeling via bi-directional 1stm-cnns-crf. In Proceedings of the 54th Annual Meeting of the Association for Computational Linguistics, 10641074. Association for Computational Linguistics.

Ma, X.; Hu, Z.; Liu, J.; Peng, N.; Neubig, G.; and Hovy, E. H. 2018. Stack-pointer networks for dependency parsing. In Proceedings of the 56th Annual Meeting of the Association for Computational Linguistics, Melbourne, Australia, July 15-20, 2018, 1403-1414.

Maier, W., and Lichte, T. 2011. Characterizing discontinuity in constituent treebanks. In Formal Grammar. 14th International Conference, FG 2009. Bordeaux, France, July 25-26, 2009.
Revised Selected Papers, volume 5591 of LNCS/LNAI, 167-182. Berlin-Heidelberg: Springer.

Maier, W., and Lichte, T. 2016. Discontinuous parsing with continuous trees. In Proceedings of the Workshop on Discontinuous Structures in Natural Language Processing, 47-57. San Diego, California: Association for Computational Linguistics.

Maier, W. 2015. Discontinuous incremental shift-reduce parsing. In Proceedings of the 53rd Annual Meeting of the Association for Computational Linguistics, 1202-1212. Beijing, China: Association for Computational Linguistics.

Martins, A.; Almeida, M.; and Smith, N. A. 2013. Turning on the turbo: Fast third-order non-projective turbo parsers. In Proceedings of the 51st Annual Meeting of the Association for Computational Linguistics, 617-622. Sofia, Bulgaria: Association for Computational Linguistics.

Müller, S. 2004. Continuous or discontinuous constituents? a comparison between syntactic analyses for constituent order and their processing systems. Research on Language and Computation 2(2):209-257.

Nivre, J. 2003. An efficient algorithm for projective dependency parsing. In Proceedings of the 8th International Workshop on Parsing Technologies (IWPT), 149-160.

Rehbein, I. 2009. Treebank-Based Grammar Acquisition for German. Ph.D. Dissertation, Dublin.

Skut, W.; Krenn, B.; Brants, T.; and Uszkoreit, H. 1997. An annotation scheme for free word order languages. In Proceedings of the Fifth Conference on Applied Natural Language Processing, ANLC '97, 88-95. Stroudsburg, PA, USA: Association for Computational Linguistics.

Stanojević, M., and G. Alhama, R. 2017. Neural discontinuous constituency parsing. In Proceedings of the 2017 Conference on Empirical Methods in Natural Language Processing, 1666-1676. Copenhagen, Denmark: Association for Computational Linguistics.

van Cranenburgh, A.; Scha, R.; and Bod, R. 2016. Data-oriented parsing with discontinuous constituents and function tags. J. Language Modelling 4:57-111.

Versley, Y. 2014. Experiments with easy-first nonprojective constituent parsing. In Proceedings of the First Joint Workshop on Statistical Parsing of Morphologically Rich Languages and Syntactic Analysis of Non-Canonical Languages, 39-53. Dublin, Ireland: Dublin City University.

Versley, Y. 2016. Discontinuity (re $)^{2}$-visited: A minimalist approach to pseudoprojective constituent parsing. In Proceedings of the Workshop on Discontinuous Structures in Natural Language Processing, 58-69. San Diego, California: Association for Computational Linguistics.

Vijay-Shanker, K.; Weir, D. J.; and Joshi, A. K. 1987. Characterizing structural descriptions produced by various grammatical formalisms. In Proceedings of the 25th Annual Meeting of the Association for Computational Linguistics, 104-111. Stanford, California, USA: Association for Computational Linguistics.

Vinyals, O.; Fortunato, M.; and Jaitly, N. 2015. Pointer networks. In Cortes, C.; Lawrence, N. D.; Lee, D. D.; Sugiyama, M.; and Garnett, R., eds., Advances in Neural Information Processing Systems 28. Curran Associates, Inc. 2692-2700.

Zhu, M.; Zhang, Y.; Chen, W.; Zhang, M.; and Zhu, J. 2013. Fast and accurate shift-reduce constituent parsing. In Proceedings of the 51st Annual Meeting of the Association for Computational Linguistics, ACL 2013, 4-9 August 2013, Sofia, Bulgaria, 434-443. 